\title{
Towards an Arabic Sign Language (ArSL) corpus for deaf drivers
}

\author{
Samah Abbas ${ }^{\text {Corresp., } 1}$, Hassanin Al-Barhamtoshy ${ }^{2}$, Fahad Alotaibi ${ }^{3}$ \\ ${ }^{1}$ Management Information Systems Department, Faculty of Economics and Administration, King Abdul Aziz University, Jeddah, Mecca, Saudi Arabia \\ 2 Information Technology Department, Faculty of Computing \& Information Technology, King Abdul Aziz University, Jeddah, Mecca, Saudi Arabia \\ 3 Information System Department, Faculty of Computing \& Information Technology, King Abdul Aziz University, Jeddah, Mecca, Saudi Arabia \\ Corresponding Author: Samah Abbas \\ Email address: sabbas@kau.edu.sa
}

Sign language is a common language that deaf people around the world use to communicate with others. However, normal people are generally not familiar with sign language (SL) and they do not need to learn their language to communicate with them in everyday life. Several technologies offer possibilities for overcoming these barriers to assisting deaf people and facilitating their active lives, including natural language processing (NLP), text understanding, machine translation, and sign language simulation. In this paper, we mainly focus on the problem faced by the deaf community in Saudi Arabia as an important member of the society that needs assistance in communicating with others, especially in the field of work as a driver. Therefore, this community needs a system that facilitates the mechanism of communication with the users using NLP that allows translating Arabic Sign Language (ArSL) into voice and vice versa. Thus, this paper aims to purplish our created dataset dictionary and ArSL corpus videos that were done in our previous work. Furthermore, we illustrate our corpus, data determination (deaf driver terminologies), dataset creation and processing in order to implement the proposed future system. Therefore, the evaluation of the dataset will be presented and simulated using two methods. First, using the evaluation of four expert signers, where the result was $10.23 \%$ WER. The second method, using Cohen's Kappa in order to evaluate the corpus of ArSL videos that was made by three signers from different regions of Saudi Arabia. We found that the agreement between signer 2 and signer 3 is $61 \%$, which is a good agreement. In our future direction, we will use the ArSL video corpus of signer 2 and signer 3 to implement ML techniques for our deaf driver system. 
2 Towards an Arabic Sign Language (ArSL) Corpus for 3 Deaf Drivers

4

5

Samah Abbas ${ }^{1}$, Hassanin Al-Barhamtoshy ${ }^{2}$, Fahad Alotaibi ${ }^{3}$

${ }^{1}$ Management Information Systems Department, Faculty of Economics and Administration, King Abdulaziz University, Jeddah, Saudi Arabia

${ }^{2}$ Information Technology Department, Faculty of Computing \& Information Technology, King Abdulaziz University, Jeddah, Saudi Arabia

${ }^{3}$ Information System Department, Faculty of Computing \& Information Technology, King Abdulaziz University, Jeddah, Saudi Arabia

Corresponding Author:

Samah A. Abbas ${ }^{1}$

Almuhammadia, Jeddah, Mecca, 23622, Saudi Arabia

Email address: sabbas@kau.edu.sa

\section{Abstract}

Sign language is a common language that deaf people around the world use to communicate with others. However, normal people are generally not familiar with sign language (SL) and they do not need to learn their language to communicate with them in everyday life. Several technologies offer possibilities for overcoming these barriers to assisting deaf people and facilitating their active lives, including natural language processing (NLP), text understanding, machine translation, and sign language simulation. In this paper, we mainly focus on the problem faced by the deaf community in Saudi Arabia as an important member of the society that needs assistance in communicating with others, especially in the field of work as a driver. Therefore, this community needs a system that facilitates the mechanism of communication with the users using NLP that allows translating Arabic Sign Language (ArSL) into voice and vice versa. Thus, this paper aims to purplish our created dataset dictionary and ArSL corpus videos that were done in our previous work. Furthermore, we illustrate our corpus, data determination (deaf driver terminologies), dataset creation and processing in order to implement the proposed future system. Therefore, the evaluation of the dataset will be presented and simulated using two methods. First, using the evaluation of four expert signers, where the result was $10.23 \%$ WER. The second method, using Cohen's Kappa in order to evaluate the corpus of ArSL videos that were made by three signers from different regions of Saudi Arabia. We found that the agreement between signer 2 and signer 3 is $61 \%$, which is a good agreement. In our future direction, we will use the ArSL video corpus of signer 2 and signer 3 to implement ML techniques for our deaf driver system. 
42

43

44

45

46

47

48

49

50

51

52

53

54

55

56

57

58

59

60

61

62

63

64

65

66

67

68

69

70

71

72

73

74

75

76

77

78

79

80

81

82

83

84

85

86

87

\section{Introduction}

Deaf people use sign language to communicate with their peers and normal people who know their sign language. Therefore, sign language is the only way to communicate with deaf people, although it receives even less attention from normal people. Furthermore, sign language is not a unified language among deaf people around the world, as each country has its own sign language. For example, American Sign Language (ASL), British Sign Language (BSL), Australian Sign Language (Auslan), Indian Sign Language (ISL) and Arabic Sign Language (ArSL). In particular, the Arab countries that use ArSL, such as the Gulf, Al-Sham, and some North African Arab countries, have similarities and differences in sign language. The reason for this is the differences between in dialects. As a result, deaf people face problems communicating with the community in many aspects while they are working or practicing their daily lives, such as health, education, and transportation. One of the solutions for these problems is using a sign language interpreter, of their work or daily life, such as health, education, and transportation. One solution to these problems is the use of a sign language interpreter, a person who knows sign language and can interpret it to normal people. However, this solution is not optimal because of loss of privacy and professional independence (Forestal,2001; Broecker,1986). Researchers have therefore developed certain technologies that allow for the provision of a computer interpreter instead of a human. For example, sign language recognition technology, machine translation (MT). In addition, some Arab researchers and organizations, such as the Arab League Educational, Cultural and Scientific Organization (ALECSO), have made efforts to unify ArSL by introducing the first dictionary in 1999 (Al-Binali\& Samareen,2009).

In Saudi Arabia, deaf people have some difficulty communicating with others. In particular, when deaf people are driving a vehicle and non-deaf people are sitting as passengers. The nondeaf passenger cannot understand the deaf driver. Also, a non-deaf passenger cannot describe the location needed by the deaf driver. However, there are many mobile applications that can be used to facilitate communication, such as Tawasol and Turjuman, but they still do not provide real-time translation (Team Mind Rockets,2017; Al-Nafjan, Al-Arifi \& Al-Wabil,2015). In addition, non-deaf passengers or drivers do not necessarily download the sign language translation application and use it only once. In the area of deaf drivers, Saudi Arabia faces a lack of technology that can improve communication between deaf drivers and non-deaf passengers. To our knowledge, no research has yet been conducted to introduce a solution in the area of deaf drivers in Saudi Arabia. In addition, there is a lack of corpus of ArSL videos being constructed in the transportation domain, especially for deaf drivers.

The purpose of this research is to publish our dataset as a continuation of previous work (Abbas, 2020). Also, we aim to publish our corpus in order to implement ML as future work. This paper is organized as follows: The second section illustrates some research work done to create videos and images of the standard corpus of sign language in each country as a database. In order to conduct their experiments to implement some systems that help deaf people in specific areas. We have also mentioned some research done in the Arab countries. In order to create an ArSL corpus by focusing on certain areas. The third section illustrates the linguistic background of ArSL, speech recognition and machine translation (MT) systems, while the fourth section focuses on the design architecture of ArSL. In the fifth section, we discuss the processing and data collection modules and evaluation videos of the deaf driver corpus that necessitated the implementation of ArSL in the deaf driving context. Finally, we illustrate future research directions.

\section{Research question}


88

89

90

91

92

93

94

95

96

97

98

99

100

101

102

103

104

105

106

107

108

109

110

111

112

113

114

115

116

117

118

119

120

121

122

123

124

125

126

127

128

129

130

131

132

133

Our research aims to answer these two questions:

1. Can we create a data corpus and video corpus for deaf drivers?

2. Can we evaluate the video corpus created of deaf drivers?

\section{Literature Review of ArSL Recognition and MT System}

This literature review illustrates various standard sign language corpora for some countries of the world. In fact, when we start designing and developing a system for deaf people, we need the essential corpora of videos or images. In addition, these corpora are considered as a reference database that must be rich with a large and complete volume of standard sign language. They will help researchers to implement machine learning techniques and develop specific systems for deaf people.

Starting with ASL, researchers developed approximately 2576 videos that included movements, hand shapes, and sentences (Martínez et al.,2002). Another researcher collected and annotated videos around a corpus of 843 sentences from Boston University and RWTH Aachen University (Dreuw et al.,2008). In Britain, approximately 249 participants completed a conversational dataset of the BSL video corpus (Schembri et al.,2018). In Indian sign language, researchers implement 1440 gestural videos of IPSL by 9 Indian signers (Kishore et al.,2011). In Brazil, they have created the LIBRAS-HCRGBDS database of the Libras video corpus which has approximately 610 videos by 5 signers (Porfirio et al.,2013). In Korean Sign Language (KSL), the researchers developed 6 thousand vocabulary words in the KSL corpus database (Kim,Jang \& Bien, 1996). In Arabic Sign Language (ArSL), 80 Arabic signers implemented a sensor-based dataset for 40 sentences (Assaleh, Shanableh \& Zourob, 2012). In addition, some researchers have produced a corpus of 500 videos and images. It contains hand shapes, facial expressions, alphabet, numbers. In addition, the movement in simple signs, continuous sentences and sentences with lip movement were performed by 10 signers. These corpuses are called SignsWorld Atlas. However, still the performed corpus does not cover all words and phrases as a database (Shohieb, Elminir \& Riad,2015). Recently, the researchers led their efforts to build the KArSL database as a comprehensive ArSL reference database for numbers, letters and words. They used Kinect devices to make these ArSL videos available to all researchers (Sidig et al., 2021).

Specifically, in ArSL, researchers are dedicating their efforts to improving the focus of accuracy in a specific domain, such as medicine and education. Some researchers used 150 video signs from the Java programming domain in their experiment (Al-Barhamtoshy et al.,2019). In the same educational field, two researchers introduced an intelligent system for deaf students based on the image. This system helped the deaf student in the educational environment. Therefore, they created the dictionary that needs it in life activities and academic environments (Mohammdi \& Elbourhamy, 2021). Some of them performed some sentences in Arabic language without focusing on a specific field. Some focused on the jurisprudence of prayer and their symbols as datasets (Almasoud \& Al-Khalifa, 2012). Other researchers used 600 phrases in the health domain (Luqman \& Mahmoud, 2019). Similarly, some researchers have implemented their research using the data created in the field of education (El, El\&El Atawy,2014; Almohimeed, Wald \& Damper,2011). In our research, we focus on a different domain that can help deaf people in the work environment, such as driving a cab. The corpus we created will help deaf drivers to communicate with non-deaf passengers while deaf people drive their cars.

\section{ArSL and Linguistic Background}

ArSL shows huge complexities in phonology, morphology, and structure, which is not the case for other sign languages. These complexities are explained below. 
134

135

136

137

138

139

140

141

142

143

144

145

146

147

148

149

150

151

152

153

154

155

156

157

158

159

160

161

162

163

164

165

166

167

168

169

170

171

172

173

174

175

176

177

178

\section{Phonology of ArSL}

Phonemes are mental representations, which is just a way to empty what is inside the brain. Phonemes are made up of four elements: 1) The shape of the hand. 2) Orientation of the hand. 3) Position of the hand. 4) Direction of the hand in motion (Schechter, 2014). In phonology, these four elements are known as hand features (MFs). In particular, in Sign Language, there are MFs and also Non-Manual Features (NMFs) are involved. NMFs refers to emotional parts of the body, for example, lip motion, facial expression, shoulder movement, head movement, eyelids and eyebrows. In general, in ArSL, we use both MFs and NMFs to give the correct meaning, called essential NMFs. On the other hand, if the signer uses just only the MFs, the meaning will turn into another meaning that the signer did not mean to express (Johnston\& Schembri, 2007).

\section{Morphology and Structure of ArSL}

The rules of grammar in ArSL are not the same as those in Arabic. The differences are: verb tenses, differences between singular and plural, rules for prepositional and adverb and gender signs. Regarding sentence structure, ArSL uses only the Subject-Verb-Object (SVO) structure instead of the SVO, OVS and VOS structures (Luqman\& Mahmoud, 2019).

\section{ArSL and Design Architecture}

New technologies that support communication have a significant impact on human life. For deaf people, the developers and researchers have tried to use some new technologies to make the life of deaf people easier by developing automated systems that can help them to communicate in various aspects of their life with others. In this section, we will illustrate the brief explanation of some of the techniques used in order to implement the automated system for better communication between the community and among themselves.

\section{Machine Translation}

Machine Translation (MT) is a standardized name for the system that relies on computer analysis to translate between two languages. It is used for text and speech using artificial intelligence (AI) and natural language processing (NLP). For example, translating from source "English" to target "Arabic" (Pradhan, Mishra\& Nayak, 2020; Verma, Srivastava\& Kumar, 2015). MT is also used to translate text or speech into video sign language or avatar. There are several approaches to MT that can be used depending on what we need to translate and what constitutes a better translation. One of these approaches is direct translation, without regard to grammar rules. To improve the quality of MT, the rule-based approach was introduced, which consists of parsing the source and target language. Another approach is corpus-based, which deals with massive data containing sentences. There are also knowledge-based approaches, which take into account the understanding of the source and target text in the context of linguistic and semantic knowledge. Finally, there is google translation which is developed by Google (Verma, Srivastava\& Kumar, 2015).

\section{Arabic Speech Recognition}

Speech Recognition (SR) is a computerized system that converts speech into text or signs. This system is used to communicate between humans and machines. It is also known as automatic speech recognition. Machine-based SR is a complicated task due to differences in dialects, contexts and speech styles. To reduce this complexity in SR, the system can exploit the repetition and structure of the token speech signal as multiple sources of knowledge. SR sources are constructed based on knowledge of phonology, syntax, phonemes, grammar and phrases (Katyal, Kaur\& Gill, 2014; Chow et al., 1987). In addition, the SR system has several classifiers. 1) The speech utterance, which is composed of separate, connected, continuous and spontaneous 
179

180

181

182

183

184

185

186

187

188

189

190

191

192

193

194

195

196

197

198

199

200

201

202

203

204

205

206

207

208

209

210

211

212

213

214

215

216

217

218

219

220

221

222

223

224

speech. 2) The speaker model, which contains one of two dependents that was designed for a specific speaker or independently for different speakers. 3) The size of vocabulary (Radha\& Vimala).

In terms of the SR process, there are four steps in which SR can be implemented. Analyze the speech signal, then extract the feature using different techniques to identify the vector, such as MFCC (Mel-frequency cepstral coefficient). Then, we build a model using different techniques like HMMs with the training dataset. In the last step, we test the model in the matching setting, taking the dissection and measuring the performance based on the error rate (Saksamudre\& Deshmukh, 2015; Yankayiş, Ensari\& Aydin) as shown in Figure 1.

\section{ArSL Gestures Recognitions}

Gesture recognition is defined as the ability of the computer to understand gestures and execute commands based on the gestures made. The first gesture recognition system was introduced in 1993 as a kind of user interface for perceptual computing that helps capture human gestures and transfer them into commands using a computerized system. It is used with many technologies, especially in the field of games, such as X-box, PlayStation, and Wii Fit. These games use Just Dance and Kinect Sports, which recognizes the hand and certain body parts (Schechter, 2014; Darrell\& Pentland, 1993).

In the sign language domain, the gesture recognition system uses the following processes: 1) Recognize the deaf signs. 2) Analyze the sign. 3) Converting that sign into meaningful text (words or phrases), voice, or expressions that non-deaf can understand. In addition, there are two main methods for gesture recognition in ArSL, namely vision-based devices (video or image) and wearable-based devices. Each of them has advantages and disadvantages. One advantage of wearable devices is that there is no need to search for background and lighting. The disadvantage is that they interfere with movement. On the other hand, the advantage of vision-based technology is the ease of movement, while the disadvantage is the effect of changing the background and lighting (Ambar et al., 2018; Paudyal et al., 2017).

In addition, each has different processes and techniques. In the vision-based process, one or more cameras are the main tools that must be available to use this method. On the other hand, the wearable-based method depends on certain types of equipment and computers mainly. In terms of process, the wearable-based method spells the alphabet by reading the specific information in each sensor of the finger or glove joint. However, the vision-based method has certain steps, which are the follows:

- Image capture, which consists in using a camera to collect data (building the corpus) and analyze the collected images.

- Pre-processing, which consists of preparing the images and identifying the information according to color (segmentation).

- Feature extraction uses some techniques like root mean square (RMS) to identify the feature vector.

Classification that classifies based on the feature vector to build the model (Mahmood\& Abdulazeez, 2019; Derpanis, 2004). The vision-based gesture recognition process is shown in the Figure 2.

\section{Deaf Driver Corpus}

For creating a deaf driver corpus, we divided the processes that we will use for this creation into 4 modules. The four modules are preprocessing, recording, assessment, and validation modules. These high-level and low-level approaches are represented in (Figure 3) and (Figure 4). 


\section{Data Collection, Creation and Annotation}

226

227

228

229

230

231

232

233

234

235

236

237

238

239

240

241

242

243

244

245

246

247

248

249

250

251

252

253

254

255

256

257

258

259

260

261

262

263

264

265

266

267

268

269

270

In this section, we explain our data collection, data determination (deaf driver terminologies) and data set creation and processing to create our video corpus through the preprocessing and recording module.

In the preprocessing module, we first collected data at two levels: a word or phrase level dataset and a sentence level dataset to create a small Arabic dictionary. This dictionary is divided into eight sections (categories). 1) Welcoming "Salam Alaikum and How are you? ". 2) Directions "Left and Right". 3) Place "School and Deaf Association ". 4) Traffic and Transportation " Driver's license and Traffic lights". 5) Sentences used by deaf drivers when they need to talk with their passengers, e.g., "We have arrived" 6) Sentences used by passengers when they need to talk with their deaf drivers, e.g., "I do not have cash to pay the amount ". 7) General Words " No and Yes and In and On ". 8) Amount "Dollar, Riyal and 2 Riyals until 100 Riyals " (Abbas, 2020).

The total words and phrases (sentences) is 215 . Some of these words and sentences fall under the general communication, collected from the Saudi Sign Language Dictionary, 2018 edition (Saudi Association for Hearing Impairment, 2018). Some of them are collected in the contextual domain of the normal conversation that was done between Saudi cab drivers and passengers. We mean in the contextual domain is each country has its own contextual domain in the payment process. For example, Saudi Arabia has its own curranty which is Riyal. Table 1 shows a part of the Arabic dictionary we created.

In the recording module, we made our videos at a rate of about 30 FPS (frames per second), by a camera for our ArSL corpus. These videos were taken by one of the ArSL expert signers who is not deaf. Figure 5 shows an ArSL corpus captured by a non-deaf expert signer. In addition to that, we made video captures with three expert signers from different occasions in Saudi Arabia. Two of them are totally deaf, while the third is hard of hearing. Figure 6 represents an ArSL corpus captured for each deaf expert. To record 215 words containing simple phrases and signs, we took about forty-five minutes continuously with the non-deaf expert signer.

However, the three deaf expert signers took approximately fifty minutes. The expert signers used only one hand if it was appropriate for the context of the deaf driver, unless the sign required the use of both hands. Next, we segmented the video using VEGAS (Video Production Software Unleash Your Creativity | VEGAS, n.d.) video editing software (we segmented each sign containing words or phrases that matched our dictionary into a single video, with a total number of 215 videos). To support future work, we added Arabic audio to each video and labeled it with Arabic text that refers to the same recorded ArSL.

\section{Dataset Evaluation}

These collected and created data will be used for the evaluation and validation of our ArSL corpus. In this section, we have explained the evaluation module while the validation will be done in the future work.

In the evaluation module, we evaluated the video of each corpus generated based on our created dictionary using two techniques. First, we used a human expert evaluation technique. Second, we used a statistical method of measuring the agreement between three deaf experts signing in ArSL that we recorded.

In the first technique, which is a human expert evaluation, we made the evaluation based on the views of four participants who are experts in ArSL. One of them is completely deaf and the second is hard of hearing. Two other participants are not deaf, they work in a deaf club and are experts. We used the quantitative approach (questionnaire) and divided it into two sections. The

Peer) Comput. Sci. reviewing PDF | (CS-2021:06:62836:1:1:NEW 26 Aug 2021) 
271 first section was a demographic questionnaire (gender - age - education level - whether he/she is

272 deaf or not). In the second section, we introduced an evaluation of the video based on the word

273 (phrase) or related sentence. We attached each video to each related phrase or word. Participants

274 were asked to evaluate the 215 sign videos to see if the video recorded for each word or phrase

275

276

277

was correct or not. If not correct, it meant that the video was not related to that particular word or phrase. We asked the four participants to choose one of the types of correction they had to make for each video (add - replace - delete). The evaluation method is explained in (Table 2) using

278 some videos evaluated by one of the experts.

279

280

The evaluation results of the deaf experts based on the word error rate (WER) for each category (section) such as welcome, directions, and place are explained in Figure 7. This means that for each category, the evaluation result of these videos was wrong. Also, they should be replaced with correct videos.

As can be seen in Figure 7, first, the hospitality category has a high percentage of WERs, at

283

284

285

286

287

288

289

290

291

292

293

294

295

296

297

298

299

300

301

302

303

304

305 $50 \%$. Second, the location category has a percentage of about $36 \%$. Thus, we need to reduce the WER in our video corpus that is related to these two categories (sections) in order to improve the communication between drivers and deaf passengers and also to describe the correct location for the passenger's destination.

The total WER of our video corpus was $10.23 \%$ as shown in Table 3 .

To resolve the corpus of erroneous ArSL videos, we again captured these videos based on the corrected signs that the raters explained to us.

In the second technique of measuring the agreement between the signs of each of the two deaf experts, we used Cohen's Kappa criterion using Eq. (1).

$$
K=\frac{\mathrm{P}_{0}-\mathrm{P}_{\mathrm{e}}}{1-\mathrm{P}_{\mathrm{e}}}
$$

Whereas $P 0$ represent the number of observed proportional agreement between two variables by using Eq. (2).

$$
\mathrm{P} 0=\frac{1}{\mathrm{n}} \sum_{i=1}^{g} f i i
$$

Number of agreements that expected by chance represented by $P e$ and the formula represents as Eq. (3).

$$
\mathrm{P}_{\mathrm{e}}=\frac{1}{\mathrm{n}^{2}} \quad \sum_{i=1}^{g} f i+f+i
$$

306

307

308

309

310

311

312

313

Where ( $(\mathrm{i}+)$ is the total of row, and $(\mathrm{f}+\mathrm{i})$ is the total of column (Viera\& Garrett,2005).

When we use Cohen's Kappa $(K)$, we can achieve one of the six types that represents in the Table 4.

For more details, the evaluation was done by a non-deaf coder who annotated each of the two ArSL video corpora on the basis of true and false independently. First, we measured the agreement between signer 1 and signer 2 . Next, we measured the agreement between signer 1 and signer 3. Finally, we measured the agreement between signer 2 and signer 3 . Therefore, by

Peer) Comput. Sci. reviewing PDF | (CS-2021:06:62836:1:1:NEW 26 Aug 2021) 
314 applying Cohen's Kappa statistical method to measure the agreement between each of the two 315 videos in the ArSL corpus, we found the result represented in Table 5.

316

317

318

319

320

321

322

323

324

325

326

327

328

329

330

331

332

333

334

335

336

337

338

339

340

341

342

343

344

345

346

347

348

349

350

351

352

353

354

355

356

357

358

359

360

361
As we can see in (Table 5) The agreement between signer 2 and signer 3 reached about $60 \%$, which is better than the others. The reason is that they are from the same school, the Deaf Association. However, signatory 1 is a volunteer who is not from their school. It should be noted that the first two are completely deaf, while the second is hard of hearing. In the validation module, in the future work, we will implement the ML technique using python as a programming language. To do this, we will use the ArSL video corpus that was made by signer 2 or signer 3 . Which one got the best agreement based on Cohen's Kappa method for evaluation. Next, we will divide our data into training and testing datasets to measure accuracy and error rate.

\section{Conclusions and Future Work}

Through this research, we have reviewed the previous studies that have been conducted in the field of ArSL corpus generation as a standard sign language used by different countries. We have also illustrated the different areas in which researchers are conducting their efforts to create a data dictionary and annotated ArSL corpus, especially in Saudi Arabia. We have clarified the difficulties encountered in translating ArSL from a grammatical, semantic and syntactic perspective. How they affect the accuracy of translation and recognition. Finally, we described the ArSL dictionary for deaf drivers and explained the data collection processes to construct the videos in our corpus. These were recorded using a single camera and then verified using two methods. First, using the evaluation of 4 participants, experts in ArSL, two of whom were deaf. Second, by using Cohen's Kappa statistical method to measure the agreement between each of the two videos in the ArSL corpus recorded by three signers.

The created ArSL corpus provides opportunities to test various feature extraction methods and recognition techniques. Extending and validating the dataset using machine learning (ML) will be the focus of future work. In addition, this corpus will be used to design our proposed system to facilitate communication with deaf drivers.

\section{References}

Abbas SA. 2020. Improving Arabic Sign Language to Support Communication between Vehicle Drivers and Passengers from Deaf People. King Abdulaziz University : Scientific Publishing Centre, Saudi Arabia.

Al-Barhamtoshy HM, Abuzinadah NE, 3, A, 4, TF, 5 and AA, Allinjawi AA. 2019. Development Of An Intelligent Arabic Text Translation Model For Deaf Students Using State Of The Art Information Technology. Bioscience Biotechnology Research Communications 12:338-345. DOI: 10.21786/bbrc/12.2/17.Al-Binali M, Samareen S. 2009. Grammar of the Unified Qatari Arabic Sign Language. Dar Al-Sharq, Doha, Qatar, In Arabic.

Alfi AEEE, Basuony MMRE, Atawy SME. Intelligent Arabic text to Arabic Sign Language Translation for Easy Deaf Communication

Almasoud AM, Al-Khalifa HS. 2012. SemSignWriting: A Proposed Semantic System for Arabic Text-to-SignWriting Translation. 2012. DOI: 10.4236/jsea.2012.58069.

Almohimeed A, Wald M, Damper RI. 2011. Arabic Text to Arabic Sign Language Translation System for the Deaf and Hearing-Impaired Community. In: Proceedings of the Second Workshop on Speech and Language Processing for Assistive Technologies. Edinburgh, Scotland, UK: Association for Computational Linguistics, 101-109.

Al-Nafjan A, Al-Arifi B, Al-Wabil A. 2015. Design and Development of an Educational Arabic Sign Language Mobile Application: Collective Impact with Tawasol. In: Antona M, Stephanidis C eds. Universal Access in Human-Computer Interaction. Access to Interaction. Lecture Notes in Computer Science. Cham: Springer International Publishing, 319-326. DOI: 10.1007/978-3-319-20681-3_30.

Ambar R, Fai CK, Wahab MHA, Jamil MMA, Ma'radzi AA. 2018. Development of a Wearable Device for Sign Language Recognition. Journal of Physics: Conference Series 1019:012017. DOI: 10.1088/17426596/1019/1/012017

Peer] Comput. Sci. reviewing PDF | (CS-2021:06:62836:1:1:NEW 26 Aug 2021) 
362

363

364

365

366

367

368

369

370

371

372

373

374

375

376

377

378

379

380

381

382

383

384

385

386

387

388

389

390

391

392

393

394

395

396

397

398

399

400

401

402

403

404

405

406

407

408

409

410

411

412

413

414

415

416

Assaleh K, Shanableh T, Zourob M. 2012. Low Complexity Classification System for Glove-Based Arabic Sign Language Recognition. In: Huang T, Zeng Z, Li C, Leung CS eds. Neural Information Processing. Lecture Notes in Computer Science. Berlin, Heidelberg: Springer, 262-268. DOI: 10.1007/978-3-642-34487-9_32.

Broecker B. 1986. Speaking the Language of Sign: The Art and Science of Signing (review). American Annals of the Deaf 131:199-200. DOI: 10.1353/aad.2012.0792.

Chow Y, Dunham M, Kimball O, Krasner M, Kubala G, Makhoul J, Price P, Roucos S, Schwartz R. 1987. BYBLOS: The BBN continuous speech recognition system. In: ICASSP '87. IEEE International Conference on Acoustics, Speech, and Signal Processing. 89-92. DOI: 10.1109/ICASSP.1987.1169748.

Derpanis K. 2004. A Review of Vision-Based Hand Gestures. Department of Computer Science York University.

Dreuw P, Neidle C, Athitsos V, Sclaroff S, Ney H. 2008. Benchmark Databases for Video-Based Automatic Sign Language Recognition. :6.

Forestal LH. A Study of Deaf Leaders' Attitudes Towards Sign Language Interpreters and Interpreting. Ph.D. Thesis. United States -- New York: New York University

Katyal A, Kaur A, Gill J. 2014. Automatic Speech Recognition: A Review. International Journal of Engineering and Advanced Technology (IJEAT) ISSN 2249-8958.

Kim J-S, Jang W, Bien Z. 1996. A dynamic gesture recognition system for the Korean sign language (KSL). IEEE Transactions on Systems, Man, and Cybernetics, Part B (Cybernetics) 26:354-359. DOI: 10.1109/3477.485888.

Kishore PVV, Kumar PR, Kumar EK, Kishore SRC. 2011. Video audio interface for recognizing gestures of indian sign. International Journal of Image Processing (IJIP) 5:479.

Luqman H, Mahmoud SA. 2019. Automatic translation of Arabic text-to-Arabic sign language. Universal Access in the Information Society 18:939-951. DOI: 10.1007/s10209-018-0622-8.

Mahmood MR, Abdulazeez AM. 2019. Different Model for Hand Gesture Recognition with a Novel Line Feature Extraction. In: 2019 International Conference on Advanced Science and Engineering (ICOASE). 52-57. DOI: 10.1109/ICOASE.2019.8723731.

Martinez AM, Wilbur RB, Shay R, Kak AC. 2002. Purdue RVL-SLLL ASL database for automatic recognition of American Sign Language. In: Proceedings. Fourth IEEE International Conference on Multimodal Interfaces. 167-172. DOI: 10.1109/ICMI.2002.1166987.

Mohammdi H, Elbourhamy D. 2021. An intelligent system to help deaf students learn Arabic Sign Language. Interactive Learning Environments. DOI: 10.1080/10494820.2021.1920431.

Porfirio AJ, Wiggers KL, Oliveira LES, Weingaertner D. 2013. LIBRAS Sign Language Hand Configuration Recognition Based on 3D Meshes. In: 2013 IEEE International Conference on Systems, Man, and Cybernetics. 1588-1593. DOI: 10.1109/SMC.2013.274.

Pradhan I, Mishra SP, Nayak AK. 2020. A Collation of Machine Translation Approaches with Exemplified Comparison of Google and Bing Translators. In: Gunjan VK, Garcia Diaz V, Cardona M, Solanki VK, Sunitha KVN eds. ICICCT 2019 - System Reliability, Quality Control, Safety, Maintenance and Management. Singapore: Springer, 854-860. DOI: 10.1007/978-981-13-8461-5_97.

Radha V, Vimala C. 2012. A review on speech recognition challenges and approaches. doaj. org 2:1-7.

Saksamudre S, Deshmukh R. 2015. Isolated Word Recognition System for Hindi Language. International Journal of Computer Sciences and Engineering 3:110-114.

Saudi Association For Hearing Impairment. 2018. Saudi Sign Language Dectionary, Riyadh.

Schechter S. 2014. What is gesture recognition? Gesture recognition defined. Available at https://www.marxentlabs.com/what-is-gesture-recognition-defined/(accessed August 4, 2021).

Schembri A, Fenlon J, Rentelis R, Reynolds S, Cormier K. 2013. Building the British Sign Language Corpus. Language Documentation \& Conservation 7:136-154.

Shohieb SM, Elminir HK, Riad AM. 2015. SignsWorld Atlas; a benchmark Arabic Sign Language database. Journal of King Saud University - Computer and Information Sciences 27:68-76. DOI: 10.1016/j.jksuci.2014.03.011.

Sidig AAI, Luqman H, Mahmoud S, Mohandes M. 2021. KArSL: Arabic Sign Language Database. ACM

Transactions on Asian and Low-Resource Language Information Processing 20:14:1-14:19. DOI:

$10.1145 / 3423420$.

Team Mind Rockets. 2017. Mind Rockets Inc, Assistive Technologies for the deaf. Available at http://mindrocketsinc.com (accessed 08 Nov 2018).

Video Production Software - Unleash Your Creativity | VEGAS. Available at https://www.vegascreativesoftware.com/au/? AffiliateID $=152 \&$ phash $=C W d 33 a e 7 \mathrm{JRr} 95 \mathrm{cbA} \& \mathrm{gclid}=C j w K C A j$ wgISIBhBfEiwALE19SU084G0eMYydNHuMFAyKgKFC8UTxNc9eZJqkoAgZENGvBd26E9EAZBoCV-

$8 Q A v D \_B w E$ (accessed July 28, 2021).

Peer] Comput. Sci. reviewing PDF | (CS-2021:06:62836:1:1:NEW 26 Aug 2021) 
417 Viera AJ, Garrett JM. 2005. Understanding Interobserver Agreement: The Kappa Statistic. Family Medicine:4.

418 YANKAYIŞ M, Ensari T, Aydin N. 2018. Performance Evaluation of Feature Extraction and Modeling Methods for 419

420 Speaker Recognition. 4.

421 
Figure 1

A diagram for recognizing spoken the Arabic language to Arabic written text (Abba, 2020)

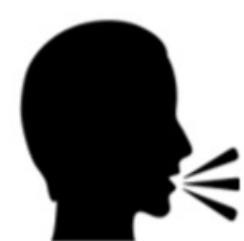

Arabic Speech Recognition

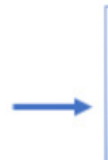

Analyzing and Segmentation
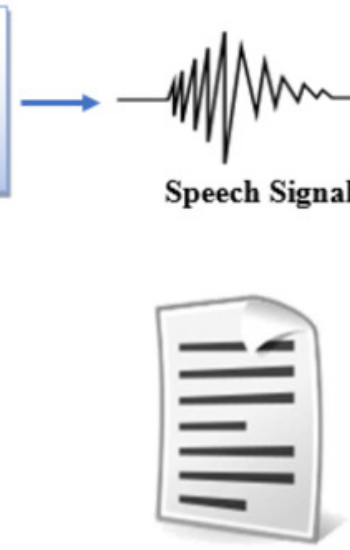

Recognized Output as Written Text

Speech Signal
Feature Extraction Using Different Techniques
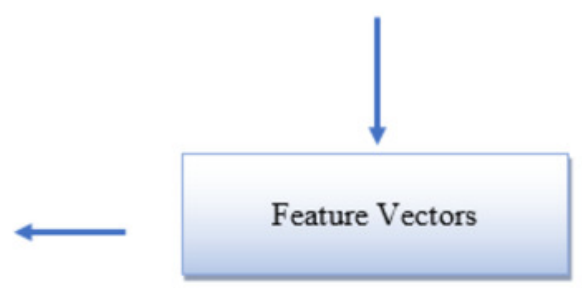
Figure 2

A diagram for the processing of ArSL gesture recognition Adapted from (Derpanis, 2004)

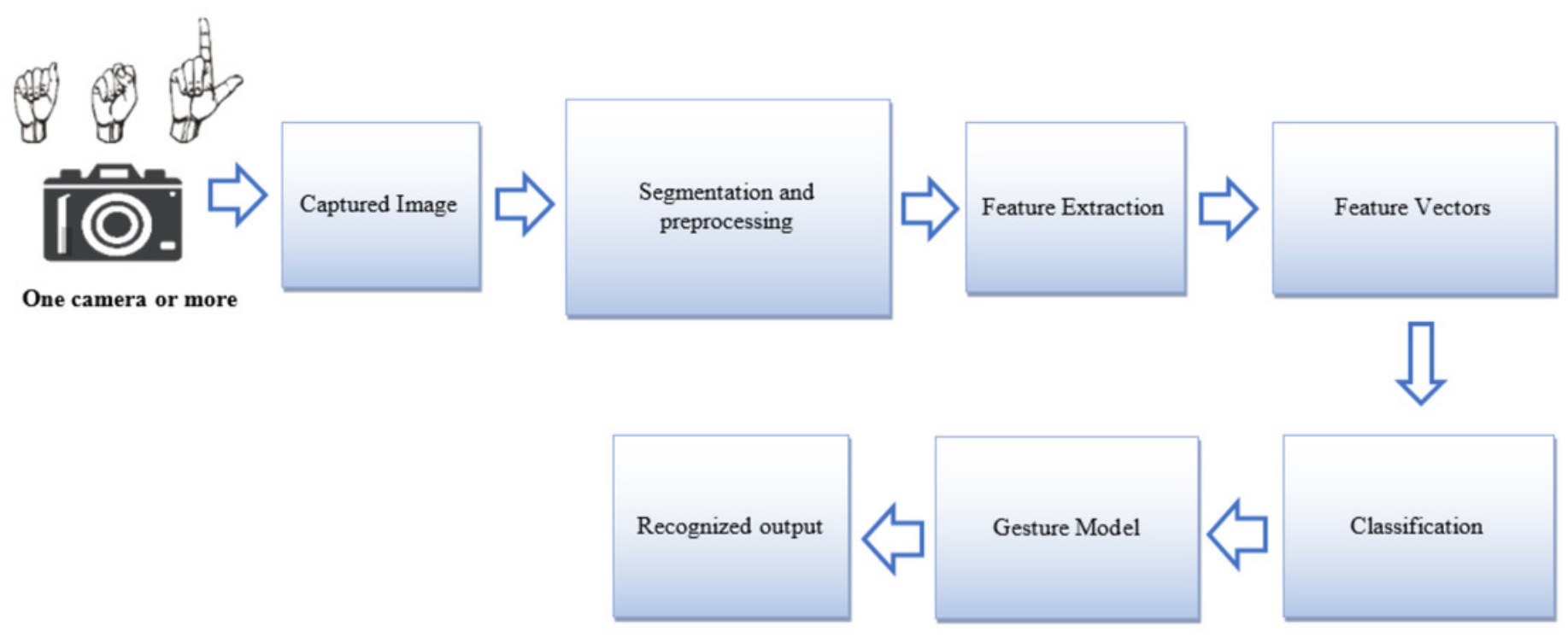




\section{Figure 3}

High-level approach of our dataset (corpus) collection (Abba, 2020)

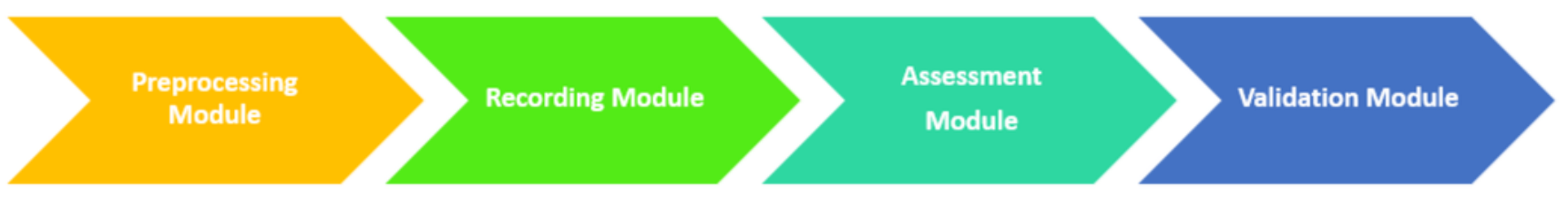




\section{Figure 4}

\section{Low-level approach of our dataset (corpus) collection (Abbas,2020)}

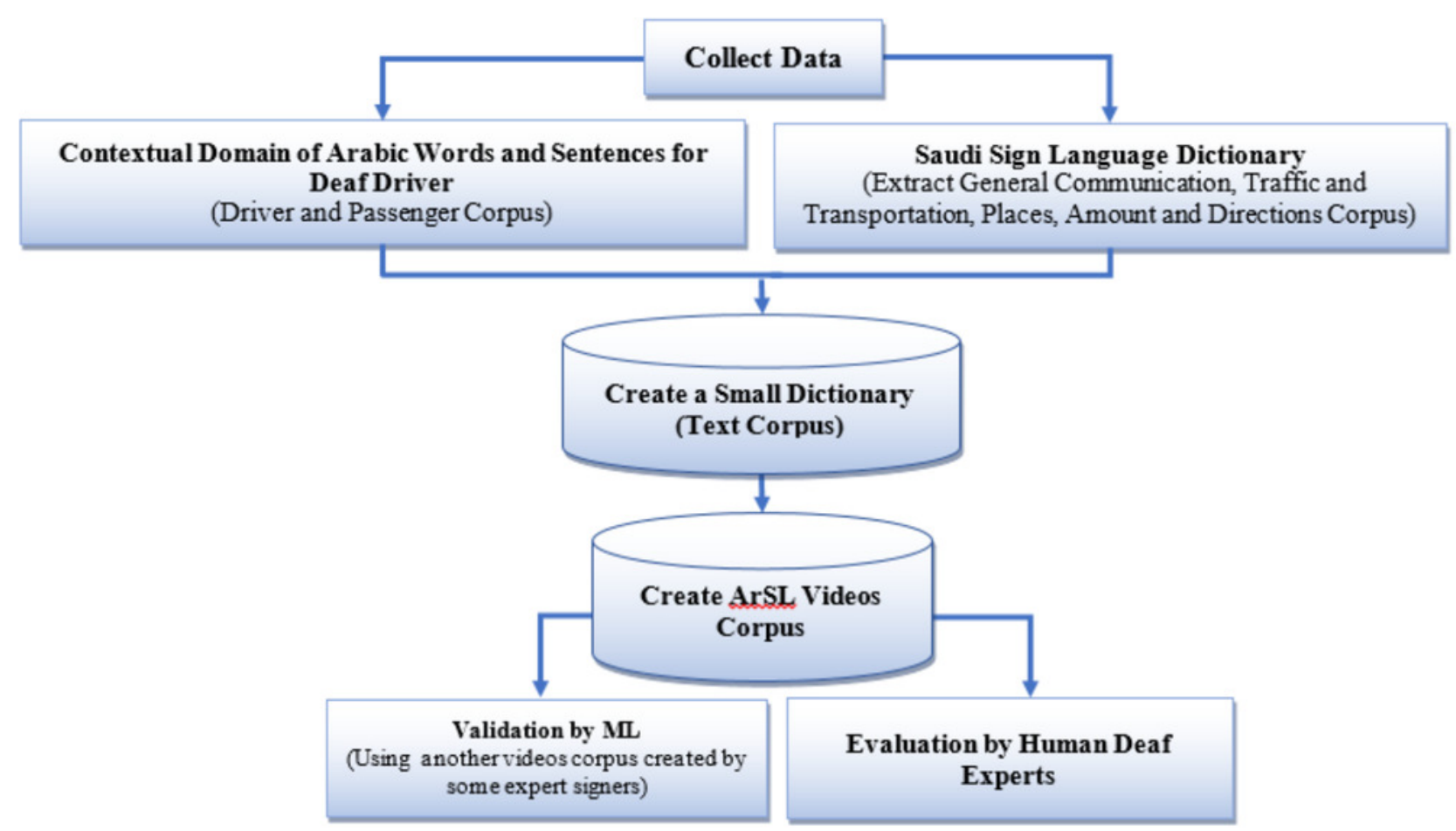




\section{Figure 5}

One Captured Video from our ArSL Video Corpus (Abbas, 2020)

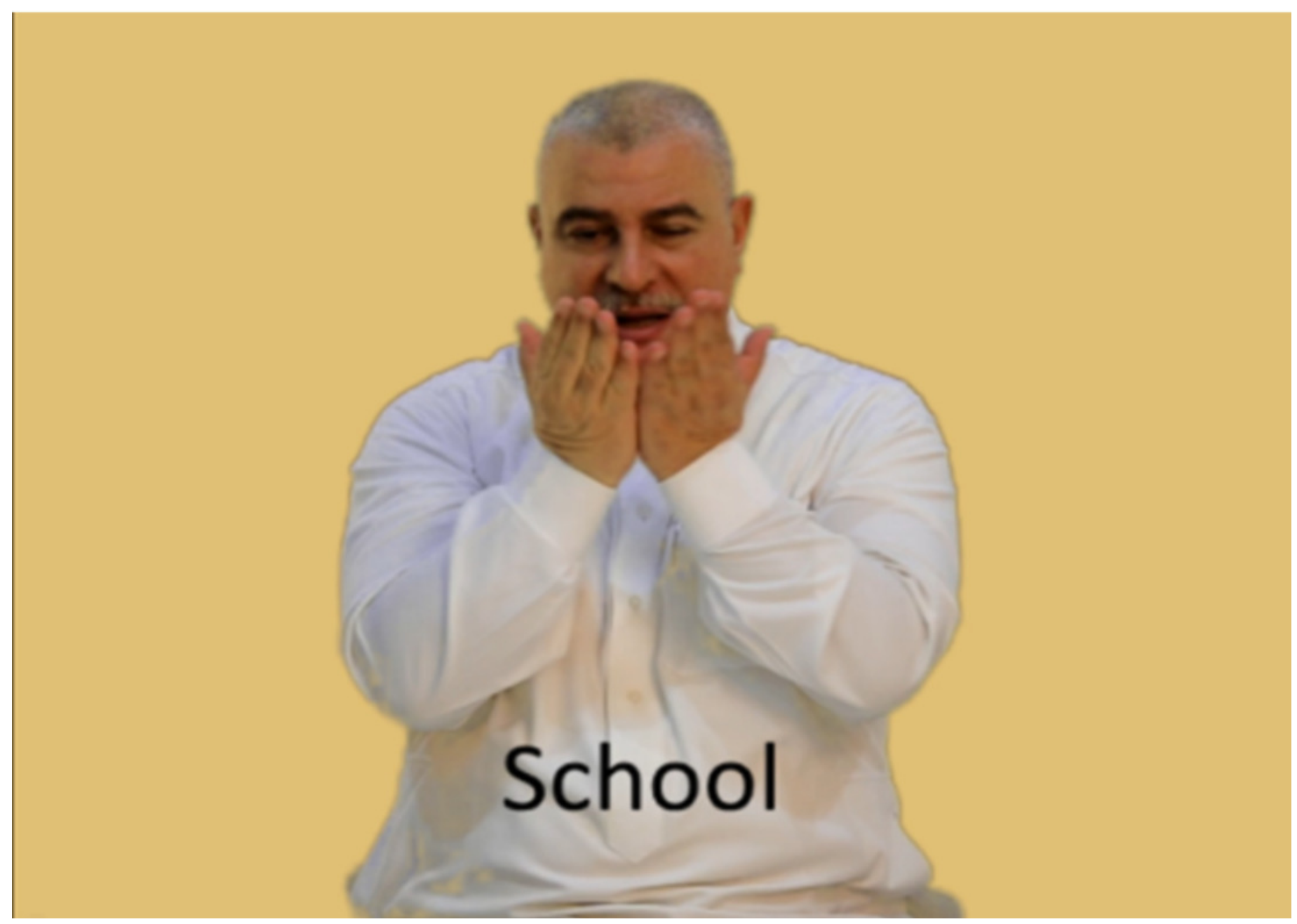




\section{Figure 6}

Three Captured ArSL Video Corpus

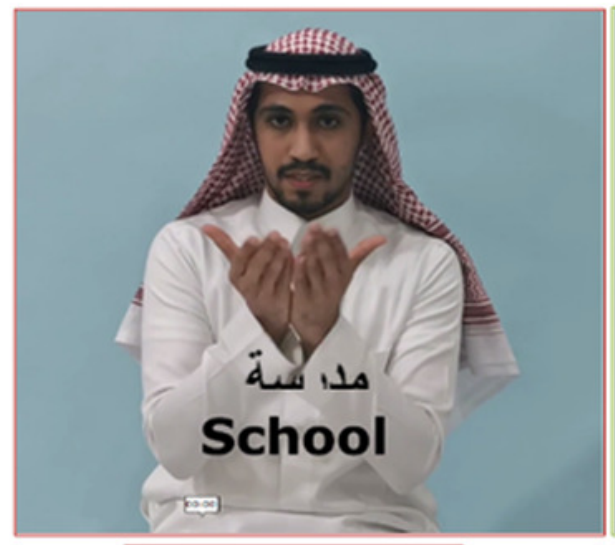

Signer1

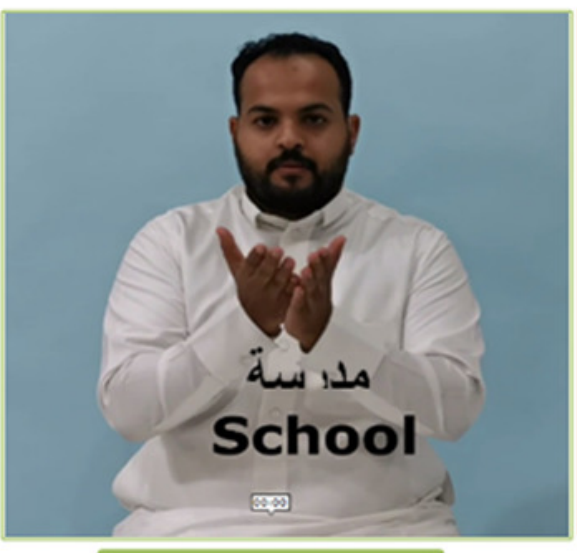

Signer2

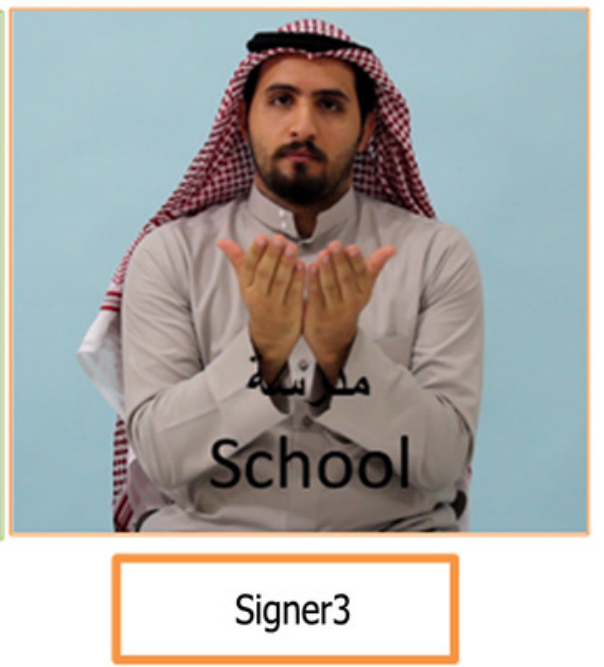




\section{Figure 7}

The Deaf Experts' Evaluations Results for Each Category Using (WER) (Abbas,2020) 


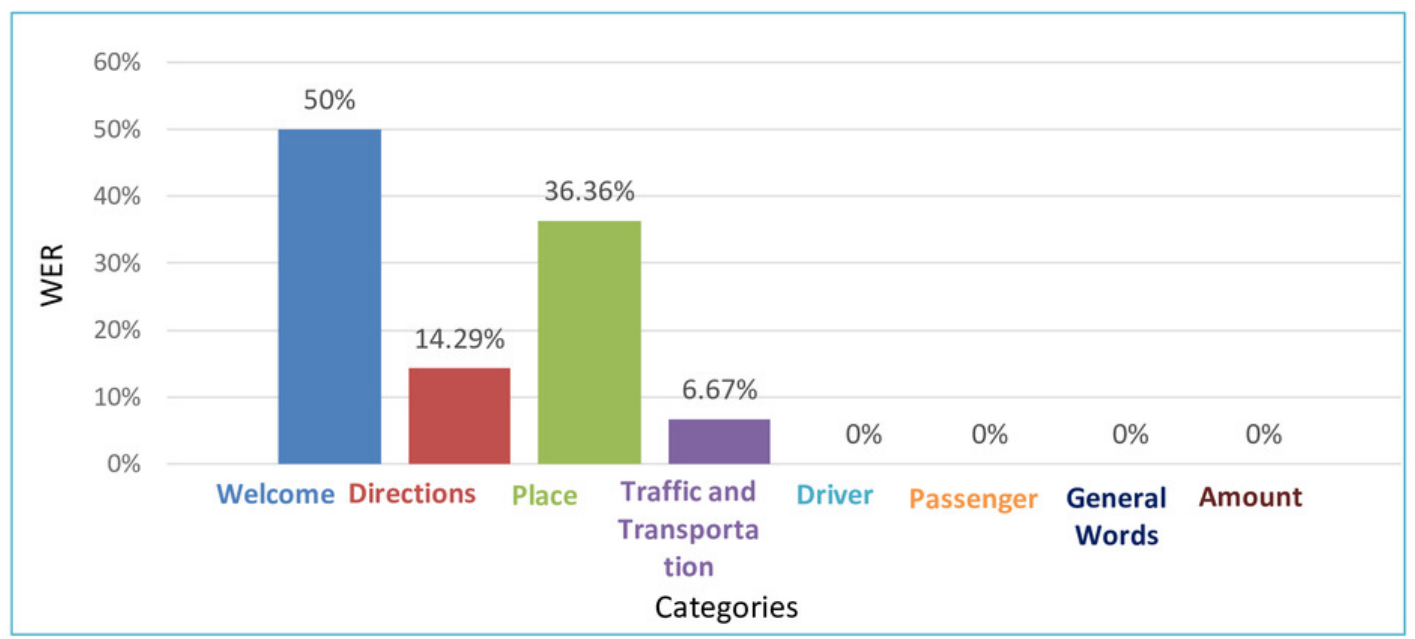




\section{Table $\mathbf{1}$ (on next page)}

An Example of our Created Dictionary (Abbas, 2020) 


\begin{tabular}{|l|}
\hline Word / Phrase / Sentences \\
\hline Section 1: Welcome \\
\hline 1.Salam Aleikum (peace be upon you) \\
\hline 2.How are you? \\
\hline Section 2: Directions \\
\hline 1. Left \\
\hline 2. Right \\
\hline Section 3: Place \\
\hline 1. School \\
\hline 2. Deaf Association \\
\hline Section 4: Traffic and Transport \\
\hline 1. Driving License \\
\hline Section 5: Driver \\
\hline 1. We have arrived \\
\hline Section 6: Passenger \\
\hline 1. I do not have cash to pay the amount \\
\hline
\end{tabular}




\section{Table 2 (on next page)}

An example of Evaluation with one Expert (Abbas,2020) 
Section One: Welcome

Correction if the translation is $\quad$ Video evaluation based on the related wrong: The video of sign needs.

\begin{tabular}{|c|c|}
\hline Deleting & Replacing \\
\hline
\end{tabular}
word (phrase) or sentences.

Word \phrase \ sentences

1-Salam aleikum

2-Waleikum salam

3-Good morning

9 


\section{Table 3 (on next page)}

Total Word Correct and Error Rate for our Created Video Corpus (Abbas,2020) 
1

2

3

4

\begin{tabular}{|c|c|c|}
\hline & Correct & Wrong \\
\hline $\begin{array}{c}\text { Signs' Videos } \\
\text { Corpus }\end{array}$ & $89.8 \%$ & $10.2 \%$ \\
\hline
\end{tabular}




\section{Table 4(on next page)}

Categories of Cohen's Kappa interpretation (Viera\&Garrett,2005) 


\begin{tabular}{|c|l|}
\hline Kappa (K) & \multicolumn{1}{|c|}{ Type of agreement } \\
\hline$K \leq 0$ & No agreement \\
\hline $0.01 \leq K \leq 0.20$ & Poor agreement \\
\hline $0.21 \leq K \leq 0.40$ & Fair agreement \\
\hline $0.41 \leq K \leq 0.60$ & Moderate agreement \\
\hline $0.61 \leq K \leq 0.80$ & Good agreement \\
\hline $0.81 \leq K \leq 1.00$ & Perfect agreement \\
\hline
\end{tabular}




\section{Table 5 (on next page)}

The Result of evaluation using Cohen's Kappa 


\begin{tabular}{|l|c|c|c|c|}
\hline & $\begin{array}{c}\text { Number of } \\
\text { observed } \\
\text { agreements }\end{array}$ & $\begin{array}{c}\text { Number of } \\
\text { agreements } \\
\text { expected by } \\
\text { chance }\end{array}$ & Kappa & Agreement \\
\hline Signer1 \& Signer2 & $78.14 \%$ & $63.97 \%$ & $39 \%$ & Fair Agreement \\
\hline Signer1 \& Signer3 & $78.14 \%$ & $66.39 \%$ & $35 \%$ & Fair Agreement \\
\hline Signer2 \& Signer3 & $\mathbf{8 7 . 9 1 \%}$ & $\mathbf{6 9 . 0 3 \%}$ & $\mathbf{6 1 \%}$ & Good Agreement \\
\hline
\end{tabular}

